\title{
Drei Fälle familiärer Hautatrophie (Poikilodermia atrophicans).
}

\author{
Von \\ Prof. Dr. Vietor Janovský.
}

(Aus dem Ambulatorium des böhmischen Kinderspitals in Prag.)

Im Jahre 1908 beschrieb Jacobi ${ }^{1}$ ) im 3. Heft der Iconographia dermatologica eine neue Hauterkrankung, welcher er den Namen Poikilodermia atrophicans vascularis beilegte.

In dem auf 2 Tafeln abgebildeten Falle handelte es sich um einen 30 jährigen, aus gesunder Familie stammenden Landwirt, welcher ohne je ernstlich erkrankt gewesen zu sein, im Jahre 1902 an Gelenkschmerzen und einer allgemeinen Müdigkeit erkrankte und bei welchem an der internen Klinik in Freiburg auf Grundlage einer ödematösen Schwellung und blaurötlichen Verfärbung der Haut im Gesicht, am behaarten Kopfe, stellenweise auch am Rumpfe und den Extremitäten die Diagnose "Sklerodermie" gestellt wurde. An der Haut waren vereinzelte narbenartige Veränderungen der Haut, Kratzeffekte durch sehr intensives Jucken verursacht und, an den Beinen flächenhafte, frischere Rötung zu sehen. Hierzu gesellte sich später noch eine ausgedehnte Fruption mit Borken besetzter Knötchen (Furunkel). Abgesehen von einer gewissen Mattigkeit fühlte sich Pat. verhältnismäBig wohl. Pat. trat dann in Behandlung der Klinik Jacobis ein. Auf die ausführliche Beschreibung Jacobis an angegebener Stelle binweisend, heben wir aus dem Status praesens kurz folgendes hervor: Auf der Kopfhaut besonders des Hinterkopfes bei dünnerem Haarwuchs etwa linsengroße atrophische Stellen, umsäumt von einem hyperämischen, teilweise bräunlich pigmentiertem Netz. Atrophie der Haut an den Ohrmuscheln. Die Gesichtshaut matt bläulichrot bis bronzegefärbt, gedunsen und ödematös, die Nasolabialfalten etwas verstrichen. Die Haut am Halse diffus gerötet mit Teleangieektasien durchsetzt stellenweise Schuppen. Die Rumpfhaut ist am auffallendsten verändert. Sie zeigt über dem Rücken in höherem Grade an der Brust in großer Ausdehnung atrophisehe Veränderungen eingerahmt von teils dunkler Pigmentierung, zahlreichen Teleangieektasien und capilläre Blutungen mit starkem Hervortreten der stecknadelkopfgroßen rotbraunen den Follikeln entsprechenden Stellen im Innern der atrophisohen Stellen. Nach Jacobi ähnelt die Affektion am meisten dem Bilde von Röntgenverbrennungen. An allen Stellen, besonders am Rumpfe, ist die Haut gespannt atrophisch, stellenweise dem Knochen anliegend. Die Schleimhaut beider Wangen zeigt ein weißliches Gitterwerk in der Ausdehnung eines Dreimarkstückes; ähn. liche Veränderungen an der den Zahnreihen anliegenden Lippenschleimhaut. Nach Jacobi entwickeln sich die atrophischen Stellen aus einem weitmaschigen, hellrot gefärbten Netzwerke, das aus Teleangieektasien und capillären Hautblutungen zusammengesetzt ist. Die Imnenorgane vollkommen gesund. Die histologische Untersuchung zeigte an den frisch erkrankten Stellen hauptsächlich eine kleinzellige Infiltration (über deren Zusammensetzung sich Jacobi nicht näher äußert) um die ektatischen Hautgefäße und um die Drüsen herum, während an den älteren Partien eine beträchtliche Atrophie aller Hautschichten, Rarefizierung des Fettgewebes, Atrophie und Zerfall der elastischen Fasern, abwechselnd Fehlen und fleckenweise Anhäufung des Pigmentes sich vorfindet. 
Jacobi differenziert hierauf die beschriebene Erkrankung von Sklerodermie, Lupus erythematosus und Erythema perstans Kaposi und spricht sich für die Eigenart seines Falles aus, indem es sich um keinen der bisher anerkannten Typen der Hautatrophie handelt. Jacobi erwähnit noch einen von Petges und Cléjat²) aus der Klinik von Dubreuilh publizierten Fall, welcher am Gesicht und Rumpf ähnliche Bilder aufweist, wo jedoch die Veränderungen an den Händen und Armen viel hochgradiger sind und wo nebstdem sich eine Myositis und Tuberkulose nachweisen ließ. Auch das histologische Bild, welches Dariers hyalin degenerierte Plasmazellen aufweist, war ein ganz anderes.

Ich teile nun im folgendem in Kürze drei Fälle einer eigentümlichen Form von Hautatrophie mit, welche zwar manche ähnliche Züge mit der von Jacobi beschriebenen Form aufweist, wobei sich jedoch die Entwicklung der atrophischen Veränderungen bloß im Gesichte lokalisiert: Die Fälle sind auch insofern interessant, als die Erkrankung familiär bei 3 Kindern einer Familie auftrat.

Die Kinder, und zwar Mädchen K. M., 12 Jahre, K. A., 10 Jahre und K. K., 8 Jahre alt, stammen aus gesunder Familie und waren mit Ausnahme von Masern, an denen sie vor längerer Zeit gelitten hatten, stets gesund. Im Herbste des Jahres 1919 erkrankten alle 3 an einer fieberhaften Erkrankung, welche der behandelnde Arzt als Influenza diagnostizierte und welche nach mehrtägiger Dauer ohne Komplikation verlief. Bald nach dieser Erkrankung bemerkte die Mutter bei allen 3 Kindern eine auffallend umgrenzte Rötung der Wangen und wie sie sich ausdrückte, eine Rauhigkeit und ein auffallendes Aussehen der Haut an den betreffenden Stellen, welches sie im November des Jahres 1919 bewog, vorerst mit ihrer 10 jährigen Tochter K. A. die Ordination des Kinderspitals aufzusuchen. Erst später brachte sie die jüngste 8 jährige und endlich die älteste 12 jährige Tochter, an welcher sie ebenfalls ähnliche Veränderungen bemerkte, in die Sprechstunde.

Vou vornherein muß bemerkt werden, dạß das Krankheitsbild am deutlichsten bei der 10 jährigen $K$. A. ausgeprägt war, während bei der jüngsten 8 jährigen Tochter die einzelnen Erscheinungen spärlicher auftraten und auf ein kleineres Territorium beschränkt blieben und bei der ältesten 12 jährigen Tochter bloß beschränkte Partien auf dem oberen Rande des streng begrenzten Herdes die charakteristischen Veränderungen aufwiesen. Um in unserer Beschreibung nicht weitläufig zu werden und um Wiederholungen zu vermeiden, beschränken wir uns bloß auf eine Schilderung des Befundes bei der 10 jährigen A. K. und wollen zum Schlusse nur ganz kurz den Unterschied im Krankheitsbilde der ältesten und jüngeren Schwester erwähnen.

A. K., 10 Jahre alt, ist ein kräftiges, ihrem Alter vollkommen entsprechend entwickeltes Kind, welches sonst vollkommen gesund ist. Auch bestehen keine Symptome, welche auf Störungen der inneren Sekretion oder auf Zirkulationsstörungen schließen ließen. Auffallend ist auf den ersten Blick bloß die Veränderung des Aussehens der Haut beider Wangen. Dieselbe ist im Umfange eines Handtellers scharf umschrieben gerötet und ganz oberflächlich infiltriert. Die Grenzen dieser Rötung, welche fast kreisförmig die Wangenhaut einnimmt, sind, wie erwähnt, scharf ausgesprochen, so dâß überall eine normale Haut die färberisch veränderten Stellen umschließt, bloß an einzelnen jedoch sehr spärlichen Stellen erscheint im geringen Umfange die Rötung sich verwaschen in die umgebende gesunde Haut zu verlieren. Die Rötung schwindet meist unter Fingerdruck 
und bloß in der Umgebung der gleich zu schildernden Veränderung bleibt nach Zurïckdrängen der Hyperämie eine unbedentende leicht gelbliche Verfärbung zurück. Das Inkarnat der roten Stellen ist ein sehr dunkles. Anzeichen von Seborrhöe sind nicht vorhanden. Die Haut fühlt sich rauh an und hat namentlich bei schief atufallendem Licht ein mattes Aussehen. Die Hornschicht verhält sich ganz normal bis auf eine unbedeutende Verdickung um die gleich zu beschreibenden Stellen. Eine Abschuppung oder ausgedehntere Verdickung des Stratum corneum ist nicht nachweisbar. Im ganzen Umfange der geschilderten Rötung erscheinen zahlreiche Einsenkungen, welche besonders dort, wo sie gedrängt beisammen stehen, der Haut ein sicbartises Aussehen vorleihen. Die Konturen dieser Einsenkungen, welche sich auf den ersten Blick als atrophische Stellen erweisen, sind verschieden, ebenso variieren dieselben bezüglich ihrer Größe. Was die letztere anbelangt, so sind dieselben oft stecknadelkopfgroß, rundlich, an anderen Stellen unregelmäßig oder irregulär polygonal, so daß stellenweise ein bienenwabenähnliches Aussehen resultiert. In diesen letzteren Fällen erscheint am Rande beim Übergange in die Umgebung an einzelnen dieser atrophischen Flecke die Hornhaut unmerklich verdickt und leicht pigmentiert. Im Grunde dieser so verschieden geformter Grübchen erscheint die Haut glatt und verdünnt. Der Finger oder Glasdruck weist in der Umgebung der atrophischen Stellen ab und zu feine GefäBreiserchen nach. Die Affektion bietet bei monatelanger Beobachtung keine Veränderung, eher eine Zunahme der atrophischen Stellen.

Die pathologischen Erscheinungen bei beiden Schwestern, der 12jährigen M. K. und der 8 jährigen $\mathbf{K}$. K. waren in betreff der primären Efflorescenzen und ihrer Anordnung dieselben. Nur die Zeit des Auftretens und die Ausbreitung der Affektion war eine versohiedene. Bei dem ältesten Mädchen (12jährig) traten die atrophisschen Stellen am spätesten auf und beschränkten sich auf eine schmale Zone am oberen Rande der streng umschriebenen Gesichtsröte, deren Farbenton auch nicht die saturierte Röte des ersten Falles hatte. Auch zeigten hier die atrophischen Stellen meist das Bild von stecknadelkopfgroßen eingezogenen Stippchen, welche selten konfluierten und die oben beschriebene wabenartige Konfiguration, wenigstens bisher, vermissen ließen. Am wenigsten ausgeprägt erscheint bisher die Affektion bei der 8 jährigen K. K. bei der bloß die scharf abgegrenzte Röte mit spärlichen etwa stecknadelkopfgroßen atrophischen Stellen sich nachweisen läßt. Verschiedene therapeutische Maßnahmen blieben erfolglos.

Eine histologische Untersuchung scheiterte an dem Widerstande der Mutter. Sie hätte wohl auch nur das aus den gründlichen Arbeiten bekannte Bild der Hautatrophien ergeben. Ein bloß auf das Gesicht beschränkter, nicht durch Sklerodermie bedingter atrophischer Prozeß ist nach den in der Literatur verzeichneten Fällen wohl selten, während in einer Reihe von Fällen bei anderweitiger Lokalisation und Ursprungs ort wir einer späteren Mitbeteiligung des Gesichtes begegnen. Am nächsten steht unseren Fällen der von Kreibich ${ }^{3}$ ) veröffentlichte Fall von Erythema perstans faciei mit Ausgang in Atrophie, wobei auch das Lippenrot eine analoge Veränderung aufwies. Freilich unterscheiden sich unsere Fälle durch die Form der stippchenartigen und stellenweise bienenwabenförmig atrophischen Stellen von dem Aussehen im Falle Kreibichs. Eben dieser ganz besondere Typus der Entwicklung und Konfiguration, die rasche Entwicklung bei ziemlich akut auftretender erythematöser mit einer oberflächlichen Schwellung verbundenen Ver- 
änderung und vor allem das familiäre Auftreten bedingt die Eigentümlichkeit unserer Fälle. Dies veranlaßte uns auch zu der Annahme, daß die Affektion dem Bilde der Poikilodermie Jacobis am nächsten steht. Seit der Veröffentlichung Jacobis sind noch einzelne Fälle beschrieben worden, von denen besonders wegen der eingehenden Beschreibung und Wertung der Befunde in differentialdiagnostischem Sinne die Arbeiten von Zinsser ${ }^{4}$ ), Glück ${ }^{5}$ ) und Müller ${ }^{6}$ ) hervorzuheben sind. Der Fall Zinssers zeigt, insofern eine Analogie mit unseren Fällen, als hier, und zwar bei dem älteren Bruder des beobachteten Falles eine analoge Erkrankung vorlag. Die Beschreibung eines ähnlichen Falles mit Lokalisation im Gesicht von Bog row ${ }^{7}$ ), dessen im Literaturberichte dieses Archivs (1907) Erwähnung geschieht, war mir nicht zugängig und scheint auch nach allem nicht in das Krankheitsbild Jacobis zu gehören. Zu den oben erwähnten Fällen von Typus Ja co bi gehört auch der von Schra me k in der Sitzung der Wiener Dermatologischen Gesellschaft der 31. X. 1912 vorgestellte Fall - Betreffs der Differentialdiagnose unserer Fälle kann Lupus erythematosus und Sklerodermie vollkommen ausgeschlossen werden, da weder die bei ersterer Erkrankung charakteristische Primärefflorescenz noch die im 2. Falle bestehende Infiltration und brettharte Verdickung nachgewiesen werden konnte. Auch für die Annahme einer Atrophia maculosa Jadassohn (Anetodermia erythematosa) lassen sich die Symptome in unseren Fällen nicht heranziehen, da namentlich die anfänglichen quaddelund papelartigen Erhebungen fehlen. Nach Jadassohns Ansicht, welche derselbe bei Demonstration eines Falles bei dem letzten Wiener Kongreß äußerte und welcher auch wir vollkommen beipflichten, würde es sich empfehlen, solche Fälle vor der Hand von den übrigen Hautatrophien abgetrennt zu behandeln und erst nach Sammlung eines größeren Materials ihre Einreihung in das System vorzunehmen.

Zum Schluß noch eine Bemerkung. Die von Zinsser und mir beobachteten familiären Fälle sind eine Stütze für die Ansicht Oppenhei $\mathrm{m} \mathrm{s}^{7}$ ), daß zur Entwicklung gewisser Arten von Hautatrophien die Annahme einer angeborenen Disposition sich nicht von der Hand weeisen läßt.

\section{Literatur.}

1) Jacobi, Poikilodermia atrophicans vascularis. Iconographia dermatologica 3. 1908. - ${ }^{2}$ ) Petges et $\mathrm{Cléjat,} \mathrm{Sclérose} \mathrm{atrophique} \mathrm{de} \mathrm{la} \mathrm{peau} \mathrm{etc.} \mathrm{myosite}$ généralisée. Ann. de dermatol. et de syphiligr. Juin 1906. - ${ }^{3}$ ) Krei bi c h, Erythema perstans mit Ausgang in Atrophie. Dieses Archiv 122. - 4) Zinsser, Atrophia cutis reticularis cum pigmentatione, dystrophia unguium et Leukoplakia oris. Iconographia dermatologica 5. 1910. -5) Glück, Dermatitis atrophicans reticularis. Dieses Archiv 118. - $\left.{ }^{6}\right) \mathrm{M}$ üller, Dieses Archiv 108. - 7) Finger und Oppenheim, Hautatrophien. 1910. 